\title{
Maíz para silo de planta entera: efecto de genotipo y altura de corte en la producción y calidad para alimentación animal
}

\author{
Victorio Morand y Celsa N. Balbi \\ Departamento de Producción Vegetal, Facultad de Ciencias Agrarias, Universidad Nacional del Nordeste, \\ Ruta 12 Km 1035. Corrientes, Argentina. (correo-e: cnbalbi@yahoo.com.ar; vicmorand516@gmail.com)
}

Recibido Ene. 7, 2020; Aceptado Mar. 9, 2020; Versión final Abr. 8, 2020, Publicado Jun. 2020

\begin{abstract}
Resumen
El objetivo de esta investigación es evaluar el efecto de genotipo y altura de corte del maíz en la producción y calidad para alimentación animal. Para evaluar tanto los materiales genéticos como el uso de ensilaje de plantas enteras se utilizarón dos híbridos de maíz (SYN 126 y SYN 139) picados a dos alturas de corte y ensilaje durante 30 días en micro silo. SYN 126 mostró más rendimiento de grano y rendimiento de silo. SYN 139 mostró una mayor digestibilidad de la materia seca y energía metabolizable. Sin embargo, la altura de corte no mostró diferencias significativas. En conclusión, el híbrido SYN 126 mostró mejor comportamiento agronómico y el híbrido SYN 139 mostró una mejor calidad nutricional. También se concluye que la decisión de la altura de corte debería pasar por la importancia de la necesidad de dejar mayor cantidad de rastrojos en el sistema productivo.
\end{abstract}

Palabras clave: forraje; digestibilidad; maíz; híbrido SYN 126; híbrido SYN 129

\section{Whole plant maize silage: effect of genotype and cutting height on production and quality of livestock feed}

\begin{abstract}
The main objective of this research study is to assess the effect of maize genotype and cutting height on the production and quality of livestock feed. In order to evaluate both the genetic materials and the use of whole plant silage, two maize hybrids (SYN 126 and SYN 139) were chopped at two cutting heights and were silage during 30 days in micro silo. SYN 126 showed higher grain and silo yield. SYN 139 showed greater dry matter digestibility and metabolizable energy. However, cutting height showed no significant differences. In conclusion, the hybrid SYN 126 had greater agronomic performance and the hybrid SYN 139 had better nutritional quality. In addition, the decision-making process on cutting heights should be examined based on the importance of the need for leaving greater amounts of stubble in the productive system.
\end{abstract}

Keywords: forage; digestibility; maize; hybrid SYN 126; hybrid SYN 129 


\section{INTRODUCCIÓN}

El cultivo de maíz en la provincia de Corrientes es relevante por su potencialidad para la alimentación animal, se utiliza tanto el grano como la planta picada en su totalidad y ensilada. La superficie de producción en Corrientes es difícil de estimar dado que muchas hectáreas de estos cultivos se derivan a la alimentación de ganado, aproximadamente existen 11 mil hectáreas sembradas de maíz en la campaña 2018/19 (Ministerio de Producción, 2019). Es una región con fuerte potencial para la siembra de maíz, lo que se viene demostrando por el aumento de superficie cultivada cada año, teniendo mayor ventaja sobre los demás granos (Arias Usandivaras, 2013). Corrientes es la cuarta en producción ganadera a nivel nacional con un stock de 4,7 millones de cabezas y tiene un perfil de gran cantidad de medianos y pequeños productores, donde el $60 \%$ del total de productores tiene menos de 100 cabezas. La ganadería bovina ocupa alrededor de 6,3 millones de hectáreas. Existen pocos estudios orientados la cadena de producción animal incluyendo la agricultura como productora de alimentos (Ministerio de Producción, 2019).

Desde el punto de vista del producto buscado, casi todos los cultivos pueden conservarse como ensilado, aunque los más empleados son los granos de gramíneas y leguminosas, y las plantas de cereales completas, especialmente trigo y maíz. El ensilado es el producto obtenido de la fermentación controlada de los cultivos de alto contenido en humedad, su proceso es llamado ensilaje. El primer objetivo, esencial para conservar los cultivos por fermentación natural, es lograr condiciones anaeróbicas. En la práctica, se consigue picando el cultivo durante la cosecha, compactando y cerrando adecuadamente el mismo. Las bacterias ácido lácticas fermentan los azúcares presentes en los cultivos (principalmente glucosa y fructosa) hasta la formación de una mezcla de ácidos, entre los que predomina el láctico (Pond et al., 2004). La superficie sembrada con maíz para silaje aumentó de manera significativa desde mediados de la década del 90 , acompañando a la creciente intensificación de los sistemas ganaderos (Arelovich et al., 2011), como consecuencia de sus múltiples ventajas tanto agronómicas como nutricionales (Carrete et al., 1997). Es así que la conservación de forraje de calidad mediante la técnica de ensilaje emerge como una alternativa valiosa en los sistemas de producción animal de nuestro país. En este sentido se busca maximizar el volumen de producción y calidad de los silajes de planta entera con el fin de lograr un producto de mejor valor nutricional y que se utilice en el bache de producción de pasturas naturales en el invierno (Arias Usandivaras, 2013).

Los tipos de silo en los que el productor ganadero puede elegir fermentar sus forrajes son muy variados, desde los pequeños sacos de plástico, hasta grandes torres cilíndricas construidas de cemento, acero o madera. Siempre que los sacos estén bien cerrados y no se perforen durante el almacenamiento, este método de conservación de hierba es totalmente adecuado (Mc Donald et al. 1995). Dentro de los criterios para elección de los híbridos a sembrar es importante tomar en cuenta el objetivo que se le dará a la producción, en este caso orientado a las necesidades nutricionales del ganado bovino (Satorre et al., 2003). Los silajes de maíz de planta entera además de aprovechar el $100 \%$ del cultivo, obtienen entre un 40 al $60 \%$ mayor rendimiento energético respecto a la cosecha del grano solamente. El maíz como tiene altos contenidos de azúcares y almidón lo que lo hace un excelente material para obtener una correcta fermentación durante el proceso de ensilado (Fisher y Burns, 1987). Además de garantizar un elevado valor nutritivo, especialmente energético que permitirá altas producciones de leche y ganancias de peso (Fernández, 2014).

La buena calidad nutricional del ensilaje de maíz como forraje y altas producciones de materia seca por unidad de superficie siempre son deseables por los productores. Giaveno y Ferrero (2003) en Santa Fe, Argentina, se encontraron que en promedio híbridos de origen tropical mostraron mayores producciones de materia seca por hectárea (22,64 $\mathrm{Tn}^{-h^{-1}}$ ) que híbridos de origen templado (19,12 Tn.ha $\left.{ }^{-1}\right)$, sobre un total de 15 materiales evaluados. Sin embargo, el mismo trabajo no encontró diferencias significativas para las variables rendimiento en granos por hectárea ni digestibilidad de la materia seca entre híbridos de origen tropical y templado; como si lo hizo para el índice de cosecha siendo menor para materiales tropicales (Giaveno y Ferrero, 2003). En México, en un ensayo con híbridos tropicales y templados no se observó que las diferencias en la digestibilidad in vitro de plantas enteras, FDN, FDA y LIGNINA estuvieran asociadas con el origen de los híbridos (Nuñez et al., 2004). También es importante la madurez a cosecha del forraje, ya que afecta la calidad del silaje de maíz porque influye sobre el contenido de humedad y la digestibilidad del resto de la planta. El estado de madurez del maíz para silaje puede determinarse por medio de la localización de la línea de leche entre 1/2 y $2 / 3$, o $35 \%$ de materia seca de la planta entera (Carrete y Scheneiter, 2012).

En lo que respecta a la altura de corte, ésta determinará para diferentes situaciones el contenido de materia seca, el cual será mayor a medida que se coseche más alto (considerando que el agua se acumula en la base del tallo); también, a medida que se eleva la plataforma de cosecha, el volumen será menor, pero se optimizará la calidad nutricional y la pureza del material (Monge y Clemente, 2014). Puede existir, además, un efecto combinado entre momento y altura de cosecha, ya que las diferencias en porcentaje de materia seca de la planta se acentúan con el avance de la madurez del cultivo (Carrete y Scheneiter, 2012). En su trabajo, Neylon y Kung (2003) observaron que pasando de una altura de corte de 12,7 cm a 45,7 cm tenían una disminución del $5 \%$ en el rendimiento de materia seca por hectárea (MS.ha-1 ${ }^{-1}$, pero con sustanciales 
aumentos en la calidad. Carrete y Scheneiter (2012) mencionan que elevar la altura de cosecha desde el nudo 1 al nudo 3, en cualquier momento de cosecha, implica disminuir entre un 4 a un $15 \%$ de forraje, entre el nudo 3 y el 5 entre un 10 y un $20 \%$ y entre el nudo 1 y el 5, entre un 20 y un $25 \%$ de forraje. Estudios realizados por Kennington et al. (2005) demostraron que pasar de una altura de corte de $20,3 \mathrm{~cm}$ a $61 \mathrm{~cm}$ mejora la composición química del silo de maíz de planta entera (\%FDN, \%FDA, \%Lignina y \%Almidón) pero no aumenta la calidad del forraje ya que no mejora la digestibilidad in situ de la MS, ni la digestibilidad in vivo de la MS.

Por otra parte, la región del noreste argentino (NEA) se caracteriza por su clima subtropical, con altas temperaturas en los meses de verano y moderados niveles de radiación solar (Maddonni, 2012). El régimen pluviométrico es isohidro con tendencia monzónica, concentrando las precipitaciones en los meses estivales (Bruniard, 2000). Debido a ésto, los cultivos son sembrados preferentemente en fechas tempranas y tardías, y no en continuo (Maddonni, 2012). Es por ello que se necesita generar información en fechas tardías. En este marco, con el entendimiento de que los futuros escenarios de producción ganadera en Corrientes tienden a unir la cadena de producción animal con la agricultura que ofrezca alimento diferido, que la expansión de la agricultura y la ganadería hacia el norte obliga a investigar la potencialidad y el comportamiento del cultivo de maíz para silaje, cantidad y calidad del alimento que se conserva; es necesario avanzar hacia la generación de información acerca del comportamiento de diferentes híbridos de maíz a distintas alturas de cortes en el ambiente productivo del NEA.

\section{MATERIALES Y MÉTODOS}

El experimento se realizó en el Campo Didáctico Experimental de la Facultad de Ciencias Agrarias de la UNNE, en la localidad de Corrientes. El sitio se encuentra ubicado en las siguientes coordenadas geográficas: Latitud: $27^{\circ} 28^{\prime 2} 27.23^{\prime \prime}$ S; Longitud: $58^{\circ} 47^{\prime} 00.66^{\prime \prime}$ O; Altitud: 50 m.s.n.m. El clima de la región se encuentra caracterizado como subtropical o templado cálido, correspondiente a los "bosques siempre verdes" de tipo mediterráneo (Bruniard, 2000). Por sus características, según Köppen, corresponde a Climas Templados Húmedos (De Fina y Ravelo, 1985; Strahler y Strahler, 1997). El suelo se encuentra clasificado como Udipsament ácuico hipertérmico de la serie Ensenada Grande (Escobar et al., 1996). Se evaluaron dos híbridos de maíz caracterizados como germoplasma tropical por templado y tropical. La densidad de siembra fue la recomendada por la empresa semillera para cada uno de los híbridos, siendo de 72.000 plantas por hectárea para SYN 126 y 55.000 plantas por hectárea para SYN 139. Se utilizaron los híbridos cuyas características agronómicas se nombran a continuación: 1) SYN 139, híbrido simple tropical ciclo completo, con tecnología Bt contra lepidópteros y coleópteros, y resistencia al herbicida glifosato; 2) SYN 126, híbrido simple tropical por templado, ciclo corto, con tecnología Bt contra lepidópteros y coleópteros, y resistencia al herbicida glifosato.

La siembra se realizó en fecha tardía el día 15 del mes de diciembre, luego de un barbecho químico con glifosato. El control de malezas, se efectuó con herbicidas pre-emergentes (atrazina y s-metolacloro) y glifosato aplicados inmediatamente posterior a la siembra. Se controlaron las plagas y enfermedades según su aparición. El cultivo se realizó sin limitantes nutricionales (lo que se logró mediante fertilizaciones periódicas con previo análisis de suelo), ni hídricas (lo que se logró a partir de riego por goteo). Las fertilizaciones sumaron un total de $200 \mathrm{~kg}$.ha ${ }^{-1}$ de $\mathrm{N}$ aplicados con urea en el riego, los demás nutrientes no fueron necesarios. Para ensilar el material vegetal se confeccionaron "micro silos" experimentales de tubos de PVC (policloruro de vinilo) de $50 \mathrm{~cm}$ de longitud y $11 \mathrm{~cm}$ de diámetro; cerrados herméticamente por sus extremos con tapas de PVC (adaptado de Laurella et al., 2017).

El picado de la planta se realizó con una corta picadora estática de la ERAGIA Corrientes y para su confección se fue compactando el material picado cargado con una prensa hidráulica, buscando una densidad de 0,8

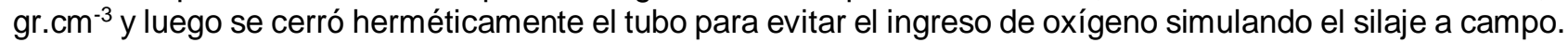
A partir los 30 días se realizaron las correspondientes mediciones de composición química. En el lote se utilizó un diseño experimental en bloques completos al azar con dos tratamientos correspondientes a dos alturas de corte de $15 \mathrm{~cm}$ (AC1) y $30 \mathrm{~cm}$ (AC2) desde el suelo; y dos materiales genéticos con cuatros repeticiones cada uno. Las parcelas se diseñaron de un tamaño de 8 surcos distanciados a $52 \mathrm{~cm}$ y $6 \mathrm{~m}$ de longitud. Las determinaciones se realizaron siempre, sobre los seis surcos centrales de cada parcela, respetando las borduras. Los análisis de muestras se realizaron a 16 microsilos correspondientes a 2 alturas de corte y 2 híbridos en las 4 repeticiones del lote.

Se registraron los estados fenológicos de los cultivos siguiendo la escala publicada por Ritchie y Hanway (1982). Se la utilizó a efectos de comparar ambos genotipos y determinar el momento de picado. Se considera que el cultivo de encuentra en fase de floración cuando los estigmas son visibles en más del $50 \%$ de las plantas (Ritchie y Hanway, 1982). Se realizaron muestreos de biomasa o MS de planta entera en R1, 15 días después y en cosecha, en dos metros lineales de surco $\left(1 \mathrm{~m}^{2}\right)$. Luego se secaron en estufa a $65^{\circ} \mathrm{C}$ hasta peso constante (aprox. 72 horas). Cada sección de la planta se pesó por separado para registro de la partición de 
asimilados y con el objetivo de calcular la tasa de crecimiento por planta (TCP) y el índice de cosecha (IC). Para registrar materia seca del picado de planta entera en media línea de leche y grano lechoso-pastoso (utilización para silo de planta entera para maíz), se cosecharon $2 \mathrm{~m}^{2}$ de cada parcela, cortando a dos alturas diferentes, $15 \mathrm{~cm}$ y $30 \mathrm{~cm}$ del suelo, eliminando las borduras (líneas laterales). Se pesó en verde y luego se secó en estufa a $65^{\circ} \mathrm{C}$ hasta peso constante para luego pesarlo nuevamente. El rendimiento final en grano se registró cuando los granos alcanzaron una humedad de aproximadamente $25 \%$, se recolectaron manualmente las espigas de un área de $1 \mathrm{~m}^{2}$ de la zona central de cada parcela para cuantificar el rendimiento final $\left(\mathrm{Kg}_{\text {.ha }}{ }^{-1}\right)$ de grano en base seca. El peso seco de grano se calculó para 1000 granos secados en estufa con circulación forzada de aire (65을 $\mathrm{C}$ ), hasta peso constante. El número de granos $\mathrm{m}^{2}$ se calculó mediante el cociente entre rendimiento de grano y el peso del grano (base seca). Los materiales picados y ya ensilados fueron analizados en el Laboratorio del CETEPRO del Ministerio de Producción de Corrientes para los siguientes parámetros químicos: Materia seca (MS) por gravimetría, Fibra detergente neutro (FDN), Fibra detergente ácido (FDA) (Van Soest et al., 1991).

Se utilizó el método de Van Soest et al. (1991) que divide los carbohidratos por su disponibilidad nutricional, describiéndolos así en forma más real y práctica desde el punto de vista de la utilidad de los carbohidratos. También se calculó la digestibilidad de la materia seca y energía metabolizable en Mcal. $\mathrm{Kg}^{-1}$ a partir de los valores de FDA; para ello se utilizaron las siguientes fórmulas: ecuación 1 (adaptado de Rohweder et al., 1978) y ecuación 2 (NRC, 1998).

DIGMS $=88,9-0,779 * \% F D A$

Energía Metabolizable $=$ DIGMS $/ 100 * 3,61$

Los datos relevados se analizaron con el software estadístico INFOSTAT (versión 2.1, Di Rienzo, 2008). Se realizó análisis de la varianza y test de Fisher para establecer diferencias entre medias de tratamientos.

\section{RESULTADOS Y DISCUSIÓN}

En la campaña 2016-2017 se sucedieron abundantes lluvias con una distribución estable a lo largo del ciclo del cultivo. Se registraron 1138,5 mm acumulados desde el 15/12/2016 hasta el 22/04/17 período entre siembra y cosecha; la máxima temperatura diaria registrada fue de $33^{\circ} \mathrm{C}$ correspondiente al día 09/01/2017, la mínima temperatura diaria registrada fue de $16,3^{\circ} \mathrm{C}$ el día 27/04/2017(Fig. 1).

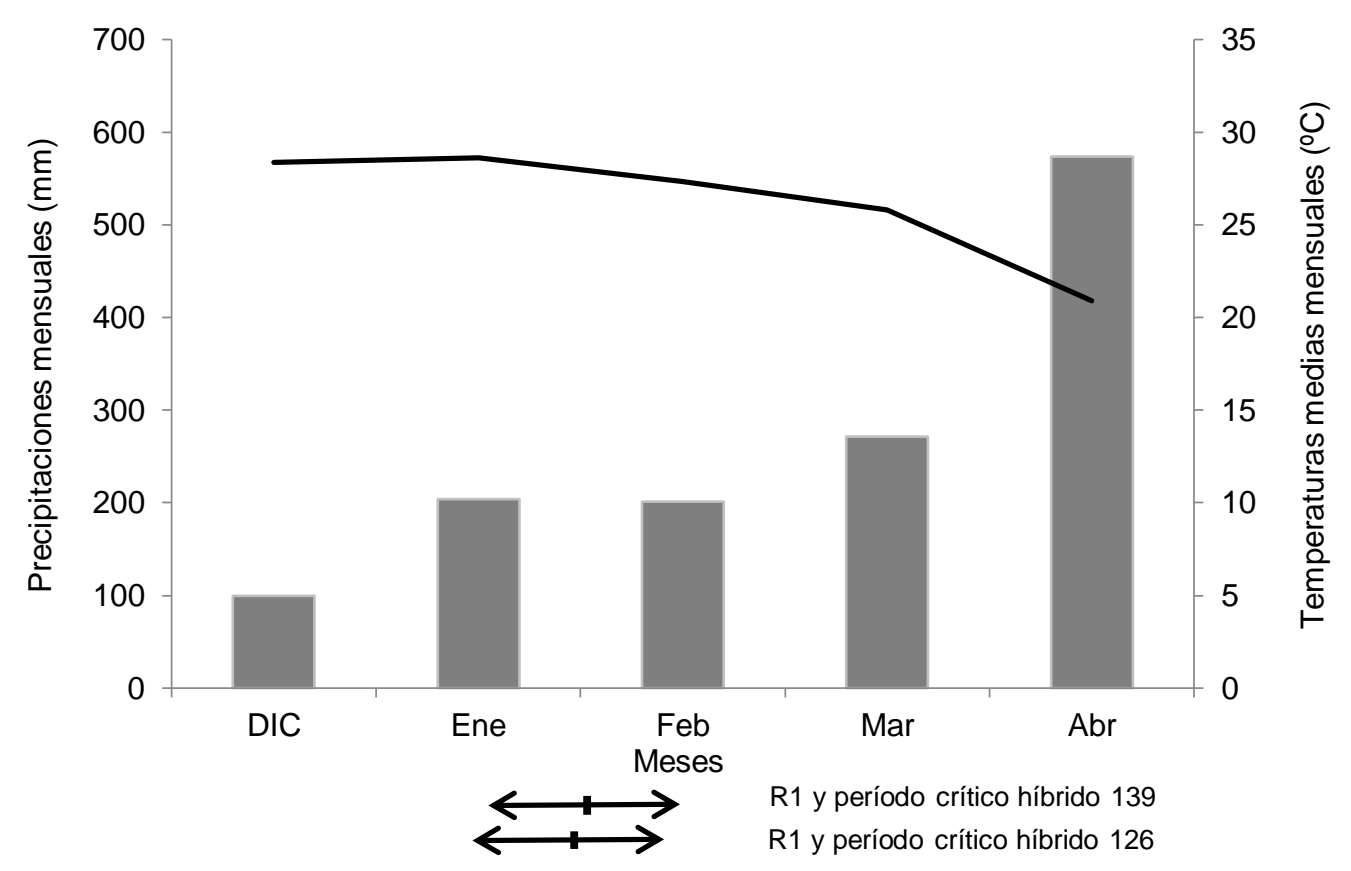

Fig. 1. Precipitaciones acumuladas mensuales (barras) y temperaturas medias mensuales (línea) a lo largo del ciclo de cultivo para la campaña 2016/2017 en Corrientes. En flechas abajo se indica la ubicación de los períodos críticos para los híbridos SYN 126 y SYN 139, la línea central indica el día de floración femenina R1. 
Respecto de la fenología del cultivo, su seguimiento se realizó siguiendo la escala de Ritchie y Hanway (1982). El tiempo térmico se calculó con temperatura base de $10^{\circ} \mathrm{C}$ (Angus et al., 1980). Se halló diferencia significativa entre ambos híbridos en lo que respecta a cantidades de días desde emergencia a floración ( $p$ valor $=0,031$ ). Siendo más precoz SYN 126 con 52,5 días promedio para las parcelas evaluadas. SYN 139 acusó un valor de 54,7 días (Tabla 1).

Un comportamiento similar se observó para los grados días acumulados a floración ( $p$-valor=0,036). El híbrido SYN 126 acumuló 1.051,4 GD a la floración y el híbrido SYN 139 1.090,2 GD. Para el período que abarca desde floración del cultivo (R1) hasta 15 días posteriores no se encontraron diferencias significativas entre híbridos ( $p$-valor=0,767). Registrándose tasas de crecimiento promedio de 4,63 g.día ${ }^{-1}$. planta $^{-1}$ para el material SYN 126 y de 4,78 g.día ${ }^{-1}$. planta ${ }^{-1}$ para SYN 139 (Tabla 2).

Tabla 1. Días desde emergencia a floración y grados días acumulados hasta R1 para dos híbridos en Corrientes. Medias con letras iguales no difieren estadísticamente ( $P$ Valor $>0,05)$.

\begin{tabular}{|l|l|l|}
\hline Híbrido & Días desde emergencia a floración & GD acumulados hasta floración \\
\hline SYN 126 & $52,50 \mathrm{~A}$ & $1.051,40 \mathrm{~A}$ \\
\hline SYN 139 & $54,70 \mathrm{~B}$ & $1.090,20 \mathrm{~B}$ \\
\hline Fuente de variación & $p$ valor & 0,036 \\
\hline HIB & 0,031 & 35,163 \\
\hline LSD (0.05) HIB & 2,177 & 1,90 \\
\hline Cv & 2,35 & \\
\hline
\end{tabular}

Tabla 2. Tasa de crecimiento por planta (TCP) para dos períodos evaluados e índice de cosecha (IC) para cada híbrido en Corrientes. Medias con letras iguales no difieren estadísticamente $(P$ Valor $>0,05)$.

\begin{tabular}{|l|l|l|l|}
\hline Híbrido & TCP R1 a R1+15 (g.día $\left.{ }^{-1}\right)$ & TCP R1+15 a Cosecha (g.día $\left.{ }^{-1}\right)$ & IC \\
\hline SYN 126 & $4,63 \mathrm{~A}$ & $1,33 \mathrm{~A}$ & $0,53 \mathrm{~A}$ \\
\hline SYN 139 & $4,78 \mathrm{~A}$ & $2,38 \mathrm{~B}$ & $0,46 \mathrm{~A}$ \\
\hline Fuente de variación & $p$ valor & 0,004 & 0,120 \\
\hline HIB & 0,767 & 0,572 & 0,088 \\
\hline LSD (0.05) HIB & 1,185 & 17,86 & 10,32 \\
\hline Cv & 14,57 & & \\
\hline
\end{tabular}

No fue así para el período comprendido entre 15 días posteriores a floración y cosecha, en el que la tasa de crecimiento por planta si arrojó diferencias significativas estadísticamente entre híbridos ( $p$-valor $=0,004)$. Siendo ésta de 1,33 g.día ${ }^{-1}$ para SYN 126 y 2,38 g.día ${ }^{-1}$ para SYN 139 (Tabla 2); diferencia a favor de éste último que podría explicarse bien por un periodo de llenado de granos más largo, por un retraso en la senescencia foliar o bien ambos; hecho que se hizo evidente a campo por permanecer el material SYN 139 más tiempo verde que SYN 126.

No se encontraron diferencias significativas entre híbridos para el índice de cosecha $(p-v a l o r=0,120)$ a diferencia de Giaveno y Ferrero (2003), quienes reportan un menor IC para materiales tropicales en el centro de Santa Fe. El híbrido SYN 126 mostró un IC promedio de 0,53 y el material SYN 139 un IC promedio de 0,46; valores similares a citados por otros autores argentinos (Andrade, 1995) para maíces graníferos templados. La diferencia entre híbridos fue significativa para rendimiento $(p$-valor $=0,005)$ y número de granos ( $p$ - valor=0,011), en contraposición a lo hallado por Giaveno y Ferrero (2003) quienes no encontraron diferencias significativas para la primera de las variables. No se hallaron diferencias significativas para el peso de los 1.000 granos ( $p$-valor=0,960). El rendimiento mayor para el híbrido SYN 126 se debió a la mayor cantidad de granos por metro cuadrado por su densidad de plantación mayor respecto al SYN 139, considerando que el peso de 1.000 granos de ambos híbridos fue similar (Tabla 3). 
Estos resultados son coincidentes con la baja plasticidad del maíz frente una disminución de densidad de plantas (Andrade y Sadras, 2002) y al hecho de que el material SYN 126 es un híbrido tropical por templado, de menor tamaño final de planta y que soporta una mayor densidad de siembra, entregando así mayor rendimiento en número de granos por hectárea respecto al material SYN 139, cuyo porte de planta es mayor.

Tabla 3. Rendimiento por hectárea, peso de 1.000 granos y número de granos por metro cuadrado para dos híbridos en Corrientes. Medias con letras iguales no difieren estadísticamente ( $P$ Valor $>0,05)$.

\begin{tabular}{|c|c|c|c|}
\hline Híbrido & Rendimiento $\left(\mathrm{Kg} \mathrm{Ha}^{-1}\right)$ & Peso 1000 granos (gramos) & Número de granos. $m^{-2}$ \\
\hline SYN 126 & $8.418,81 \mathrm{~A}$ & $280,63 \mathrm{~A}$ & $3.017,75 \mathrm{~A}$ \\
\hline SYN 139 & $6.061,51 \mathrm{~B}$ & $281,25 \mathrm{~A}$ & $2.155,50 \mathrm{~B}$ \\
\hline Fuente de variación & \multicolumn{3}{|l|}{$p$ valor } \\
\hline HIB & 0,005 & 0,960 & 0,011 \\
\hline LSD (0.05) HIB & $1.332,310$ & 29,000 & 587,040 \\
\hline Cv & 10,64 & 5,97 & 13,12 \\
\hline
\end{tabular}

\section{Rendimiento y humedad de silo}

A diferencia de lo hallado por Giaveno y Ferrero (2003) en Santa Fe donde híbridos de origen tropical mostraron mayor producción de MS que materiales templados en el presente trabajo el rendimiento de silo medido en $\mathrm{Kg}$ de materia seca por hectárea (Tabla 4) fue significativamente mayor para el híbrido templado SYN 126 ( $p$-valor $<0,001$ ), probablemente debido a su mayor densidad de siembra, comportamiento que se condice con el de la variable rendimiento en grano analizada anteriormente (Tabla 3).

Tabla 4. Rendimiento de silo en MS por hectárea y humedad del material al momento del picado para dos híbridos a dos alturas de corte distintas en Corrientes. Medias con letras iguales no difieren estadísticamente ( $P$ Valor $>0,05)$.

\begin{tabular}{|l|l|l|l|}
\hline Híbrido & Altura de corte & Rendimiento de silo $\left(\mathrm{Kg} \mathrm{MS.} \mathrm{ha}^{-1}\right)$ & Humedad (\%) \\
\hline \multirow{2}{*}{ YN 126 } & AC1 & $24.39 \mathrm{~A}$ & $67,25 \mathrm{~A}$ \\
\cline { 2 - 4 } & AC2 & $23.54 \mathrm{~A}$ & $68,00 \mathrm{~A}$ \\
\hline \multirow{2}{*}{ SYN 139 } & AC1 & $9.72 \mathrm{~B}$ & $73,75 \mathrm{~B}$ \\
\cline { 2 - 4 } & AC2 & $10.16 \mathrm{~B}$ & $74,75 \mathrm{~B}$ \\
\hline Fuente de variación & $p$ valor & \\
\hline HIB & $<0,001$ & 0,001 \\
\hline TRAT & 0,887 & 0,809 \\
\hline HIB X TRAT & 0,658 & 0,109 \\
\hline LSD $(0.05)$ HIB & $3.093,918$ & 1,101 \\
\hline LSD $(0.05)$ TRAT & $3.093,918$ & 1,101 \\
\hline LSD $(0.05)$ HIB X TRAT & $4.375,461$ & 1,557 \\
\hline CV & 16,75 & 1,42 \\
\hline
\end{tabular}

Respecto de la humedad del picado de planta entera, se encontraron diferencias entre híbridos ( $p$ valor $<0,001)$, siendo menor para el material SYN 126 probablemente por ser este de ciclo más corto con énfasis en el llenado de granos. Considerándose que éste alcanzó la floración 2,2 días promedio antes que SYN 139 y que el picado se realizó 5 días antes para lograr la humedad de silo buscada, la mayor humedad de SYN 139 puede deberse a una fase de llenado de granos más larga de este último o estar correlacionado con su mayor capacidad para mantenerse verde observada a campo en este ensayo respecto a SYN 126. 
También cabe aclarar que, si bien la media línea de leche fue el criterio adoptado a campo para establecer el momento óptimo de picado para ambos híbridos por igual, el material SYN 139 acumuló un 25,75\%MS en promedio al momento del corte, valor menor que el 32,38\%MS de SYN 126. Ésta diferencia posiblemente esté asociada a factores genéticos.

No se encontraron diferencias estadísticas en el rendimiento del silo para los tratamientos "alturas de corte" en ninguno de los dos materiales evaluados; a diferencia de lo hallado por Neylon y Kung (2003) que afirman que pasar de la altura de corte de $12,7 \mathrm{~cm}$ a $45,7 \mathrm{~cm}$ puede reducir el rendimiento del forraje un $5 \%$; y lo hallado por Carrete y Schneiter (2012) que reportan importantes pérdidas de rendimiento en MS al aumentar la altura de corte. Probablemente esto se deba a que la diferencia de altura de corte de los tratamientos planteada en el presente trabajo (de más de la mitad de la utilizada por Neylon y Kung) no fue suficiente para provocar cambios significativos estadísticamente en la producción de MS por hectárea. Tampoco se encontraron diferencias estadísticas en el porcentaje de humedad para los tratamientos "alturas de corte" como si reportan Monge y Clemente (2014). Dado que la diferencia en altura entre el corte a $15 \mathrm{~cm}$ y $30 \mathrm{~cm}$ no fueron suficientes para generar cambios significativos en el contenido de humedad y rendimiento del silo para las condiciones de cultivo del presente ensayo, investigación posterior será necesaria para evaluar el efecto de mayores diferencias de altura de corte o bajo distintas condiciones de cultivo.

En lo que respecta a la calidad nutricional del ensilaje, no se encontraron diferencias significativas para los tratamientos altura de corte en ninguno de los dos híbridos ensayados para ninguna de las variables evaluadas (Tabla 5); a diferencia de lo encontrado por Kennington et al. (2005) en sus trabajos donde alturas de corte de $20,3 \mathrm{~cm}$ y $61 \mathrm{~cm}$ arrojaron diferencias significativas en el \%FDN y \%FDA; aunque no hallaron diferencias en la digestibilidad de la materia seca para las AC mencionadas.

Tabla 5. Calidad nutricional del material ensilado según híbrido y altura de corte (AC). Medias con letras iguales no difieren estadísticamente ( $P$ Valor $>0,05)$.

\begin{tabular}{|c|c|c|c|c|c|}
\hline Híbrido & Altura de corte & $F D N(\%)$ & $F D A(\%)$ & $\begin{array}{l}\text { Digestibilidad } \\
\text { de la MS (\%) }\end{array}$ & 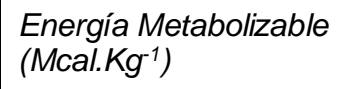 \\
\hline \multirow[t]{2}{*}{ SYN 126} & $\mathrm{AC} 1$ & $46,11 \mathrm{~A}$ & $29,42 \mathrm{~A}$ & $65,99 \mathrm{~A}$ & $2,38 \mathrm{~A}$ \\
\hline & AC2 & $44,72 \mathrm{~A}$ & $27,95 \mathrm{~A}$ & $67,13 \mathrm{~A}$ & $2,42 \mathrm{~A}$ \\
\hline \multirow[t]{2}{*}{ SYN 139} & $\mathrm{AC} 1$ & $58,02 \mathrm{~B}$ & $23,95 \mathrm{~A}$ & $70,24 \mathrm{~A}$ & $2,54 \mathrm{~A}$ \\
\hline & AC2 & $56,56 \mathrm{~B}$ & $23,33 \mathrm{~A}$ & $70,73 \mathrm{~A}$ & $2,55 \mathrm{~A}$ \\
\hline \multicolumn{2}{|c|}{ Fuente de variación } & \multicolumn{4}{|l|}{$p$ valor } \\
\hline \multicolumn{2}{|l|}{ HIB } & 0,001 & 0,026 & 0,026 & 0,026 \\
\hline \multicolumn{2}{|l|}{ TRAT } & 0,587 & 0,609 & 0,608 & 0,610 \\
\hline \multicolumn{2}{|c|}{ HIB X TRAT } & 0,989 & 0,836 & 0,836 & 0,840 \\
\hline \multicolumn{2}{|c|}{ LSD (0.05) HIB } & 5,531 & 4,326 & 3,370 & 0,122 \\
\hline \multicolumn{2}{|c|}{ LSD (0.05) TRAT } & 5,531 & 4,326 & 3,370 & 0,122 \\
\hline \multicolumn{2}{|c|}{ LSD (0.05) HIB X TRAT } & 7,822 & 6,119 & 4,766 & 0,172 \\
\hline \multicolumn{2}{|l|}{$\mathrm{Cv}$} & 9,89 & 15,18 & 4,51 & 4,52 \\
\hline
\end{tabular}

En contraste con lo encontrado por Nuñez et al. (2004) en México donde parámetros de calidad no estuvieron asociados al origen del germoplasma, el \%FDN mostró diferencias significativas entre los silos de los distintos híbridos ( $p$-valor $=0,001$ ) y el \%FDA mostró un comportamiento similar a la variable anterior ( $p$-valor $=0,026$ ); el \%FDN fue inferior para el híbrido SYN 126 y el \%FDA mostró un comportamiento opuesto, siendo menor para el híbrido SYN 139 (Tabla 5). En Santa Fe, Argentina, Giaveno y Ferrero (2003) no encontraron diferencias en la digestibilidad de la comparación de materiales, lo que puede deberse a que en este trabajo el híbrido utilizado en vez de ser puro templado se trata de una cruza con tropical, el cual esta mas adaptado a la región NEA. Este comportamiento, sumado a la diferencia de humedad puede deberse una lignificación de los tejidos mucho más lenta en el híbrido SYN 139 o bien que este proceso de deposición de la misma se dé más avanzado el ciclo fenológico o bien no se dé nunca. 
Si bien el híbrido SYN 139 posee un mayor porcentaje de fibra detergente neutro, su menor porcentaje de fibra en detergente ácido permite dilucidar qué gran porcentaje de esta fibra corresponde a hemicelulosa, su parte más digestible (Van Soest y Wine, 1967). Y a esto se debe el hecho de que la digestibilidad de la materia seca arroje valores mayores para el híbrido SYN 139. A diferencia de lo encontrado por Nuñez et al. (2004) en su trabajo, donde la digestibilidad in vitro de plantas enteras no estuvo asociada al origen del germoplasma, en este ensayo la Digestibilidad de la Materia Seca mostró diferencias significativas entre híbridos ( $p$-valor= 0,026 ) siendo la media de $66,56 \%$ para el material SYN 126 y de $70,48 \%$ para SYN 139 . Es por esto último que la Energía Metabolizable también mostró una diferencia significativa para los distintos híbridos, siendo la media para el SYN 126 de 2,4 Mcal. $\mathrm{Kg}^{-1}$ y para el SYN 139 de 2,54 Mcal. $\mathrm{Kg}^{-1}$. Ello podría deberse a las diferencias en partición a órganos (láminas, vainas, tallos y granos) en diferentes híbridos (Sun et al., 2009).

\section{CONCLUSIONES}

De acuerdo al trabajo presentado y a los resultados obtenidos, se pueden plantear las siguientes conclusiones principales:

El híbrido SYN 126, de ciclo más corto en dos días a R1, mostró mejor comportamiento agronómico logrado principalmente por una mayor producción total de biomasa y un mayor número de granos por metro cuadrado dado que su menor porte permite una mayor densidad de siembra.

Por otra parte, el híbrido SYN 139 mostró una mejor calidad nutricional ya que los valores de FDA fueron menores que los del híbrido SYN 126. Es por este mismo motivo que los datos de EM y Digestibilidad calculados fueron mayores en el híbrido SYN 139.

Respecto de las alturas de corte, no se encontraron diferencias en rendimiento de silo ni en calidad nutricional en ningún material, con lo cual la decisión de la altura de corte debería pasar por la importancia de la necesidad de dejar mayor cantidad de rastrojos en el sistema productivo.

\section{REFERENCIAS}

Arelovich, H.M., Bravo R.D. y Martínez M.F., Development, Characteristics, and Trends for beef Cattle Production in Argentina. https://doi.org/10.2527/af.2011-0021, Animal Frontiers, 1 (2), 37-45 (2011)

Andrade, F.H., Analysis of Growth and Yield of Maize, Sunflower and Soybean grown at Balcarce, Argentina. Field Crops Research 41.1 1-12 (1995)

Angus, J. F., Cunningham R. B. y otros dos autores, Phasic Development in Field Crops I. Thermal Response in the Seedling Phase. https://doi.org/10.1016/0378-4290(80)90042-8, Field Crops Research, 3, 365-378 (1980).

Arias Usandivaras, L., Elección de Híbridos, Fertilización y Densidad de Siembra en Maíz en Lomadas Arenosas de Corrientes, Revista Noticias y Comentarios, 502 (2013).

Bruniard, E., Los Regímenes Climáticos y la Vegetación Natural Aportes para un Modelo Fitoclimático Mundial, Publicación Especial (16), 79 (2000).

Carrete, J. R. y Scheneiter O., Maíz para Silaje; en Bases para el Manejo del Cultivo de Maíz por G. H. Eyhérabide, 219234, Ediciones INTA, Pergamino, Argentina (2012).

Carrete, J., Scheneiter J., Rimieri P. y Devito C., Maíz para Silaje: Efecto del Momento de Cosecha sobre la Producción y el Valor Nutritivo del Forraje. Revista de Tecnología Agropecuaria, 2(6), 2-5 (1997).

De Fina, A. y Ravelo C. A. Climatología y Fenología Agrícolas, 4a Ed., 351 p., EUDEBA, Buenos Aires, Argentina (1985).

Di Rienzo, J., Casanoves F. y otros cuatro autores, InfoStat, versión 2008, Universidad Nacional de Córdoba, Córdoba, Argentina (2008).

Echarte, L., y Andrade F. H., Harvest index stability of Argentinean Maize Hybrids released between 1965 and 1993. Field Crops Research 82.1 1-12 (2003).

Escobar, E., Ligier D. y otros tres autores, Mapa de Suelos de los Departamentos de Capital, San Cosme e Itatí de la Provincia de Corrientes, Argentina. Publicación del Convenio del INTA-ICA y Provincia de Corrientes-CFI, 129 (1996).

Fernández Mayer, A., Impacto de los Silajes de Planta Entera (Maíz o Sorgo) en los Sistemas de Engorde Intensivos (Pastoril y a Corral). Recuperado el 11 de Octubre de 2017, de Instituto Nacional de Tecnología: http://www.inta.gob.ar (2014).

Fisher, D. S. y Burns J. C. Quality Analysis of Summer-Annual Forages. II. Effects of Forage Carbohydrate Constituents on Silage Fermentation. https://doi.org/10.2134/agronj1987.00021962007900020014x, Agronomy Journal, 79(2), 242-248 (1987).

Giaveno, J. y Ferrero C., Introduction of Tropical Maize Genotypes to Increase Silage Production in the Central Area of Santa Fe, Argentina. https://doi.org/10.12702/1984-7033.v03n02a01, Crop Breeding and Applied Biotechnology, 3(2), 8994 (2003). 
Kennington, L. R., Hunt C. W. y otros tres autores, Effect of Cutting Height and Genetics on Composition, Intake, and Digestibility of Corn Silage by Beef Heifers. https://doi.org/10.2527/2005.8361445x, Journal of Animal Science, 83(6), 1445-1454 (2005).

Laurella, E. D., Jovtis M. L. y otros cinco autores, Composición Química Comparativa entre Ensilaje de Cebada y Centeno, Revista Argentina de Producción Animal, 37(1), 344 (2017).

Maddonni, G. A., Analysis of the Climatic Constraints to Maize Production in the Current Agricultural Region of Argentina, a Probabilistic Approach. https://doi.org/10.1007/s00704-011-0478-9, Theoretical and Applied Climatology, 107, 325-345 (2012).

Mc Donald, P., Edward y R. y Greenhalgh J. F., Nutrición Animal, 5 a Ed., Acribia, Madrid, España (1995).

Ministerio de Producción de Corrientes. Recuperado el 06 de Julio de 2019, http://www.mptt.gov.ar (2019).

Monge, J. L. y Clemente G., Calidad de silaje, $5^{\circ}$ Jornada Nacional de Forrajes Conservados, Recopilación de Presentaciones Técnicas, Ediciones INTA, 139-144, Manfredi, Argentina (2014).

Neylon, J. M. y Kung L., Effects of Cutting Height and Maturity on the Nutritive Value of Corn Silage for Lactating Cows. https://doi.org/10.3168/jds.S0022-0302(03)73806-5, Journal of Dairy Science, 86(6), 2163-2169 (2003).

Nuñez, H. G., Contreras G. F. y Faz C. R., Producción, Composición Química y Digestibilidad In Vitro de Híbridos de Maíz de Origen Tropical y Templado en la Región Semiárida de México, ISSN: 0188-7890, Avances en Investigación Agropecuaria, 8(1) (2004).

Pond, W. G., Church, D. B., Pond, K. R. y Schoknecht, P. A. Basic animal nutrition and feeding. John Wiley \& Sons. (2004)

Ritchie, S. W., y Hanway J. J., How a Corn Plant Develops, Special Report N48, lowa State University of Science and Technology, Cooperative Extension Service (1982).

Rohweder, D. A., Barnes R. F. y Jorgensen N., Proposed Hay Grading Standards Based on Laboratory Analyses for Evaluating Quality. https://doi.org/10.2527/jas1978.473747x, Journal Animal Science, 47, 747-759 (1978).

Satorre, E. H., Benech Arnold R. y otros cinco autores, Producción de Granos: Bases Funcionales para su Manejo, $1^{\text {a }}$ Ed., EUDEBA, Buenos Aires, Argentina (2003).

Strahler, A. N. y Strahler A. H., Geografía Física, 3a Ed., OMEGA, Barcelona, España (1997).

Sun, Z.H., Liu, S.M., Tayo, G.O. y otros ocho autores. Effects of cellulase or lactic acid bacteria on silage fermentation and in vitro gas production of several morphological fractions of maize stover. https://doi.org/10.1016/j.anifeedsci.2009.04.013. Animal Feed Science and Technology, 152(3-4), 219-231. (2009)

Van Soest, P. J. y Wine R. H., Use of Detergents in the Analysis of Fibrous Feeds. IV. Determination of Plant Cell-wall Constituents, Journal of the association of Official Analytical Chemist 50(1), 50-55 (1967).

Van Soest, P. J., Robertson J. B. y Lewis B. A., Methods for Dietary Fiber, Neutral Detergent Fiber, and Nonstarch Polysaccharides in Relation to Animal Nutrition. https://doi.org/10.3168/jds.S0022-0302(91)78551-2, Journal of Dairy Science, 74(10), 3583-3597 (1991). 
